\title{
Genetic potential of tropical sweet corn hybrids and combining ability among parental inbred lines
}

\author{
Neyaz Rashid Mustafa ${ }^{1}$, Ghizan Bin Saleh ${ }^{1 *}$, Pedram Kashiani ${ }^{2}$ \\ ${ }^{1}$ Department of Crop Science, Faculty of Agriculture, Universiti Putra Malaysia, 43400 Serdang, Selangor, \\ Malaysia \\ ${ }^{2}$ Department of Agriculture Science, Faculty of Technical and Vocational Education, Universiti Pendidikan \\ Sultan Idris, 35900 Tanjung Malim, Perak, Malaysia
}

*Corresponding author: ghizan@upm.edu.my

\begin{abstract}
Superiority of sweet corn (Zea mays L. saccharata) hybrid varieties is reflected by their performance per se, heterosis they reveal and combining ability of their inbred parents. This study was conducted to investigate performance, combining ability, heterosis and heritability revealed by $15 \mathrm{~F}_{1}$ tropical sweet corn hybrids from a half-diallel cross involving six diverse inbred lines, at two locations in Malaysia. At each location, the hybrids were evaluated for ear yield and yield components in a randomised complete block design, in comparison with their inbred parents and a commercial hybrid variety Hybrid 530 as control. Hybrids $\mathrm{H} 11, \mathrm{H} 12$ and $\mathrm{H} 15$ were found to be the most superior for yield and yield components, hence could be further tested in large-scale trials before release. Inbred lines FTT-1, EEO-2 and HAW-1 showed high positive GCA effects for yield and yield-related traits at both locations. Cross combinations HAV- $2 \times$ NTS2, HSE-4 $\times$ NTS-2, NTS-2 $\times$ EEO-2 and EEO-2 $\times$ HAW-1 revealed high positive SCA effects for fresh ear yield and yield related traits at both location. Crosses among unrelated lines were found to produce superior hybrids. Both additive and non-additive gene effects were found important, although additive effects were predominating. The hybrids revealed substantially high heterosis, the highest being for number of ears per hectare and ear height. Moderate to high broad- and narrow-sense heritabi1ity estimates were displayed by the different traits measured.
\end{abstract}

Keywords: diallel analysis; gene effects; heritability; heterosis; Zea mays L. saccharata.

Abbreviations: ANOVA_Analysis of variance; BPH_Better-parent heterosis; GCA_General combining ability; MPH_Mid-parent heterosis; SAS_Statistical Analysis System; SCA_Specific combining ability.

Introduction

Development of sweet corn (Zea mays L. saccharata) hybrid varieties involves selection of superior inbred lines, hybridization, evaluation of hybrid performance and selection of best crosses to manifest hybrid vigour. Hybrid varieties display high vigour and plant uniformity. Hybrid vigour or heterosis has been described as the ability of hybrid progenies to outperform their parents for a particular trait (Falconer and Mackay, 1996). It is essential for hybrids to express high heterosis, which is usually influenced by the degree of dominance and differences in gene frequencies (Hallauer and Miranda, 1988). Its expression is partly dependent on genetic diversity between the parental lines used, which could be in the form of increased yield and dry matter accumulation, earliness in maturity, increased uniformity, improved quality and increased resistance to pests and diseases.
In the advanced stage of inbred line selection, sweet corn breeders determine the best combination between selected inbred lines in relation to other available lines (Burt et al., 2011). General combining ability (GCA) and specific combining ability (SCA) are important parameters that give the potential merit of inbred lines to be utilized in hybrid combinations. Variations in GCA effects have been associated with additive genetic variance and the interaction of additive and additive variance, while variations in SCA effects have been associated with non-additive genetic variance (Falconer and Mackay, 1996). Estimation of combining ability can be determined through performance evaluation on all possible crosses (diallel cross), where the merit of each inbred line can be evaluated. A diallel analysis provides good information on the genetic identity of genotypes, specifically on dominance-recessive relationships and some other genetic associations (Ahmad et al., 2009). Information on the magnitude of important genetic variances in the population 
contributing to high heterosis and combining ability is required to enable effective selection for yield and other desirable traits (Vacaro et al., 2002). This information helps researchers to develop appropriate selection techniques and to evaluate heterotic patterns at the initial stage of hybrid breeding programs (Le Gouis et al., 2002).

This study was conducted to evaluate performance of tropical single-cross sweet corn hybrids obtained from crosses among six selected inbred lines, to estimate general and specific combining abilities among the parental inbred lines involved, to estimate heterosis exhibited by the hybrids, and to estimate heritability of the traits measured on the hybrid populations.

\section{Results}

\section{Performance of hybrids}

The effects of genotypes were significant $(p<0.05)$ for all 10 traits measured in both locations. In general, differences were noted on the performance among the genotypes evaluated, for all traits measured in Field 2 and Field 10 (Tables 2 and 3, respectively). In Field 2, the hybrids had fresh ear yields lower than that of the control variety, Hybrid 530 (26285 kg ha-1). However, in Field 10, their fresh ear yields were comparable to that of Hybrid 530 (19179 $\left.\mathrm{kg} \mathrm{ha}^{-1}\right)$. For kernel total soluble solids (TSS) concentration in Field 2, performances of the hybrids were comparable to that of Hybrid 530 (15.9\% Brix). In Field 10, many hybrids even produced higher kernel TSS than Hybrid 530 (16.7\% Brix). At both locations, H4, H13, H14 and H15 consistently produced high ear lengths, with mean values of 20.4, 20.0, 19.6 and $20.8 \mathrm{~cm}$, respectively in Field 2 and 19.6, 20.6, 19.0 and $20.9 \mathrm{~cm}$, respectively in Field 10. Furthermore, in Field 2, H12 produced the highest ear diameter $(49.4 \mathrm{~mm})$ among the hybrids, which was comparable to that of Hybrid 530 $(49.0 \mathrm{~mm})$. However, in Field $10, \mathrm{H} 11, \mathrm{H} 12$ and $\mathrm{H} 15$ revealed the highest ear diameters (46.3, 47.4 and $47.2 \mathrm{~mm}$ respectively), which were higher than that of Hybrid $530(42.8 \mathrm{~mm})$.

\section{General and specific combining abilities of inbred lines}

Mean squares from ANOVA on the $6 \times 6$ diallel cross hybrids involving the sweet corn inbred lines (Griffing's Method 4, Model I) for the 10 traits measured are presented in Tables 4 and 5 for data from Field 2 and Field 10, respectively. The effects of hybrids were significant for all traits at both locations, indicating the presence of high variations among them. Consequently, variations due to hybrids were partitioned into GCA and SCA effects.

The estimates of GCA effects on the parental inbred lines are shown in Table 6. GCA effects on fresh ear yield varied among the six parental inbred lines at both locations. Inbred lines FTT1, EEO-2 and HAW-1 had high positive GCA effects for fresh ear yield, with estimates of 1415.89, 1132.22 and 777.89, respectively, in Field 2, and 975.22, 1321.89 and 1204.89, respectively, in Field 10 . This indicates that they were effective general combiners, with good accumulation of additive genes for fresh ear yield. In Field 2, NTS-2 and EEO-2 had positive GCA effects for TSS ( 0.88 and 0.15 , respectively), whereas HSE-4 and HAW- 1 had negative GCA effects for TSS $(-0.68$ and -0.28 , respectively). In Field 10 however, only NTS- 2 showed a positive GCA effect for TSS (1.01). All parental inbred lines appeared to have high GCA effects $(p<0.01)$ for ear length in Field 2. In Field 10 however, only EEO-2 and HAW-1 showed positive GCA effects for ear length (1.06 and 0.96, respectively). Moreover, FTT-1 had positive GCA effects for ear diameter at both locations (2.64 in Field 2 and 1.65 in Field 10).

Regarding SCA effects for fresh ear yield in Field 2, the highest estimates were revealed by H3 (HAV- $2 \times$ NTS- 2 ), H13 (NTS- $2 \times$ EEO-2) and H15 (EEO-2 $\times$ HAW-1), with positive effects of 2399.53, 2827.47 and 1012.20, respectively (Table 7). In Field 10, only H7 (HSE-4 $\times$ NTS-2) had positive SCA effects for fresh ear yield (1746.47). The inbred parents of these hybrids were found to be from diverse source populations. In Field 2, the highest estimate of SCA effects for TSS was shown by H1 (HAV$2 \times$ HSE-4) (0.74). However, in Field 10, H7 (HSE-4 $\times$ NTS-2) was found to possess the highest SCA effects for TSS (2.25). In Field 2 , the highest estimates of SCA effects for ear length were shown by H3 (HAV-2 $\times$ NTS-2), H12 (FTT-1 $\times$ HAW-1), H13 (NTS$2 \times$ EEO-2) and H15 (EEO-2 $\times$ HAW-1), with estimates of 1.91, $1.39,1.68$ and 0.87 , respectively, while the lowest estimate were exhibited by $\mathrm{H} 2$ (HAV-2 $\times$ FTT-1) (-1.58), H9 (HSE-4 $\times$ HAW1) $(-0.86)$ and H14 (NTS-2 $\times$ HAW-1) $(-0.75)$. However, in Field 10, H4 (NTS-2 $\times$ HAW-1) and H7 (HSE-4 $\times$ NTS-2) were found to possess the highest SCA effects for ear length (0.89 and 1.08, respectively), whereas $\mathrm{H} 1$ (HAV-2 $\times$ HSE-4) showed the lowest estimate $(-0.83)$. In Field 2, positive SCA effects for ear diameter were exhibited by H10 (FTT- $1 \times$ NTS-2) (5.91) and H13 (NTS- $2 \times$ EE0-2) (4.35), but negative SCA effects were revealed by $\mathrm{H} 2$ $(\mathrm{HAV}-2 \times \mathrm{FTT}-1)(-2.56)$ and H6 (HSE-4 $\times$ FTT-1) $(-2.41)$. In contrast, in Field 10, positive SCA effects for ear diameter were only revealed by H4 (HAV-2 × EEO-2) (2.69).

High GCA to SCA ratios were observed for most of the traits measured (Table 8). This emphasizes the fact that additive gene effects were more prevailing in the control the traits compared to the non-additive counterpart. Negative estimates of GCA to SCA ratio were also observed for some traits, due to negative variances, and therefore considered as zero.

\section{Heterosis and heritability}

In general, estimates of heterosis (Table 8) were positive for all traits measured, with the exceptions of better-parent heterosis (BPH) for number of ears per hectare at both locations $(-10.3 \%$ and $-3.1 \%$, respectively in Field 2 and Field 10$), \mathrm{BPH}$ for ear height in Field $10(-0.8 \%)$, and mid-parent heterosis (MPH) and $\mathrm{BPH}$ for days to tasseling and silking at both locations $(-7.1 \%$ for $\mathrm{MPH}$ and $-8.7 \%$ for $\mathrm{BPH}$ for days to tasseling, and $-6.4 \%$ for MPH and $-8.0 \%$ for BPH for days to silking, in Field 2, and $-6.6 \%$ for $\mathrm{MPH}$ and $-8.5 \%$ for $\mathrm{BPH}$ for days to tasseling, and $-5.8 \%$ for $\mathrm{MPH}$ and $\mathbf{- 7 . 7 \%}$ for BPH for days to silking, in Field 10). For days to tasseling and silking however, these negative values of heterosis are favorable because they indicate that the hybrids flowered earlier than their parents.

In general, moderate to high broad-sense $\left(\mathrm{h}^{2} \mathrm{~B}\right)$ and narrowsense heritability $\left(\mathrm{h}^{2} \mathrm{~N}\right)$ were observed for the traits measured (Table 8). In Field 2, broad-sense heritability estimates ranged from $25.95 \%$ (days to silking) to $91.18 \%$ (ear height), while in Field 10, they ranged from $18.43 \%$ (ear diameter) to $49.99 \%$ (ear height). For narrow-sense heritability however, estimates ranged from $8.50 \%$ (ear diameter) to $76.59 \%$ (TSS) in Field 2, and from $22.04 \%$ (days to silking) to $75.71 \%$ (number of ears per hectare) in Field 10. 


\section{Discussion}

The hybrids revealed substantial amount of variation for all traits measured at both locations, indicating the presence of a high genetic variability among them for the genes they carry. Among the new hybrids evaluated, $\mathrm{H} 11, \mathrm{H} 12$ and $\mathrm{H} 15$ consistently exhibited high performance for almost all traits measured at both locations. These high yielding hybrids were found to be from crosses between inbred lines that were combinations of high $\times$ high, high $\times$ low or low $\times$ low yielding inbred lines from diverse source populations. Past workers have also reported significant variations in agronomic performance among diverse sweet corn hybrids evaluated in different environments (Revilla et al., 2005; Kashiani et al., 2011; Suzukawa et al., 2018).

GCA and SCA effects were significant for all traits measured on the 15 sweet corn hybrids at both locations, indicating the substantial contribution of both additive and non-additive gene actions in the genetic expression of the traits concerned. Findings from past studies have also emphasized on importance of GCA and SCA effects in genetic control of sweet corn traits (Dhasarathan et al., 2015; Dermail et al., 2019). Inbred lines FTT1, EEO-2 and HAW-1 consistently showed their superiority by revealing high positive GCA effects for fresh ear yield, ear weight, ear length, diameter, plant height and ear height at each location. This was verified by the fact that the hybrids produced from these inbred lines gave high yield and yield-related traits at both locations. In contrast, inbred lines HAV-2, HSE-4 and NTS-2 were the poor combiners because they exhibited negative GCA effects for most traits where positive values were favoured. This finding suggests the presence of a substantial diversity in the genetic constitutions of the parents involved for most of the traits studied. It was also found that, in general, inbred lines with high GCA effects for yield had moderate to high GCA effects for most of the yield components. In contrary, inbred lines with high GCA for yield components did not necessarily have high GCA for yield. Not all high yielding cross combinations at each location showed high and positive SCA effects. Similarly, hybrids that revealed high SCA effects did not necessarily include all those that gave high performance per se. Among the new hybrids evaluated, consistently high SCA effects for fresh ear yield, ear weight, ear length, ear diameter, plant height, ear height, days to tasseling and days to silking were expressed by H3 (HAV-2 $\times$ NTS- 2 ) and H13 (NTS- $2 \times$ HAW- 1 ) in Field 2, and H7 (HSE-4 $\times$ NTS-2) in Field 10. Although NTS-2, which was the common parent of these hybrids, showed negative GCA effects for fresh ear yield, ear weight, ear length, ear diameter, plant height and ear height, significant positive SCA effects and high heterosis were detected in hybrids involving this inbred line. Hybrids $\mathrm{H} 3, \mathrm{H} 13$ and $\mathrm{H} 7$ were products from inbred lines with high $\times$ high, high $\times$ low or low $\times$ low SCA. In addition, $\mathrm{H} 15$ which was the progeny of the cross between EEO-2 and HAW-1, constantly gave high performance, high heterosis and high SCA effects at both locations, for fresh ear yield, weight, ear length, ear diameter, plant height, ear height, days to tasseling and days to silking. It is therefore suggested that $\mathrm{H} 15$ is further utilized in crosses for good exploitation of heterosis in breeding programs. The predominant expression of specific combining ability for ear diameter, sugar content, carbohydrate accumulation pattern in kernels and ear length have also been previously described (Bordallo et al., 2005; Ha, 1999; Khanduri et al., 2010; Rovaris et al., 2017.

The high GCA to SCA variance ratios for fresh ear yield, number of ears per hectare, TSS, ear length, ear diameter, ear height, and days to tasseling observed at each location imply that, although additive and non-additive gene actions were both important in the control of these traits, additive gene action was more prevalent. The GCA to SCA variance ratios varied with traits and locations, as have also been previously reported (Kumar et al., 2019).

As expected, all hybrids were more superior than their respective parental lines for almost all traits studied, justifying for the presence of substantial amount of heterosis. Mid- and better-parent heterosis estimates obtained were positive for all traits measured except days to tasseling and days to silking, where the lower magnitudes were favoured, indicating earliness. In a study on sweet and waxy corn hybrids derived from 24 single crosses, evaluation at two seasons in Thailand found that MPH and BPH for fresh ear yield ranged from $67.36 \%$ to $252.60 \%$, and from $18.59 \%$ to $183.56 \%$, respectively (Dermail et al., 2019). This indicates that, to exploit heterosis, selection of suitable parents is essential.

With regards to broad-sense heritability, which is the ratio of the total genetic variance to the phenotypic variance (Falconer and Mackay, 1996), moderate to high estimates were noted for all traits measured at both locations, with the exceptions of ear diameter and days to silking in Field 10, which showed low estimates (18.43 and $19.84 \%$, respectively). This implies that the expressions of these sweet corn plant traits were sufficiently heritable. Moderate to high broad-sense heritability estimates for all traits measured in sweet corn population crosses were reported (Ali et al., 2003), while high broad-sense heritability estimates for plant height, number of days to tasseling and ear length in sweet corn synthetic populations were also observed (Saleh et al., 2002). A high broad-sense heritability for TSS in super sweet corn populations was also noted (Yao et al., 2018). In terms of narrow-sense heritability, which is the ratio of additive variance to phenotypic variance (Falconer and Mackay, 1996), high estimates were observed for most of the important traits which include fresh ear yield, number of ears per hectare, ear weight, ear length and ear height at both locations, indicating that additive genetic component was responsible for the phenotypic variability observed among the hybrids. This gives the reflection that additive genetic effects were important in the control of these traits, implying that sweet corn hybrid breeders could improve important traits by accumulating dominant genes in the diverse inbred lines they develop. Moderate to high broad-sense heritability estimates for traits measured in seven sweet corn varieties were reported (Alan et al., 2013), while low narrowsense heritability estimates for all traits studied were also documented (Sujiprihati et al., 2003; Azad et al., 2014). Low estimates of narrow-sense heritability indicate that many genes were involved in the regulation of the traits, allowing high influence of the environmental factors (Suzuki et al., 1981). The influence of interaction between genetic and environmental factors was also reflected to a lesser extent by moderate magnitude of narrow-sense heritability observed for TSS concentration in super sweet corn populations (Yao et al., 2018). 
Table 1. Six sweet corn parental inbred lines utilized in the half-diallel cross, and a control variety used in the study.

\begin{tabular}{|l|l|c|}
\hline Inbred line / variety & $\mathrm{S}_{6}$ & Level of selfed generation \\
\hline HAV-2 & $\mathrm{S}_{6}$ & Hybrid Harvest \\
\hline HSE-4 & $\mathrm{S}_{6}$ & Hybrid H7 \\
\hline FTT-1 & $\mathrm{S}_{6}$ & Hybrid 522 \\
\hline NTS-2 & $\mathrm{S}_{6}$ & Hybrid 926 \\
\hline EEO-2 & $\mathrm{S}_{6}$ & Hybrid 8800 \\
\hline HAW-1 & $\mathrm{F}_{1}$ & Hybrid Hawaiian \\
\hline Hybrid 530 (Control) & & Commercial hybrid \\
\hline
\end{tabular}

Table 2. Mean values for traits measured on 15 sweet corn hybrids, six parental inbred lines and a control variety (Hybrid 530 ), evaluated in Field 2.

\begin{tabular}{|c|c|c|c|c|c|c|c|c|c|c|}
\hline \multirow[t]{2}{*}{ Genotype } & \multicolumn{10}{|l|}{$\operatorname{Mean}^{a}$} \\
\hline & FEY & $\mathrm{NE}$ & EW & TSS & EL & ED & $\mathrm{PH}$ & $\mathrm{EH}$ & DT & DS \\
\hline \multicolumn{11}{|l|}{ Hybrid: } \\
\hline $\mathrm{H} 1$ (HAV-2 $\times$ HSE-4) & $17960 \mathrm{fg}$ & 45226 fghi & $335.8 \mathrm{fg}$ & $16.1 \mathrm{abcd}$ & $17.9 \mathrm{gh}$ & 47.2 cde & 171.4 ef & 86.6 de & $43.8 \mathrm{defg}$ & $45.0 \mathrm{cdefg}$ \\
\hline H2 (HAV-2 × FTT-1) & 18675defg & 67413 a & 349.2 defg & 15.8 bcde & $18.3 \mathrm{fg}$ & $48.3 \mathrm{abc}$ & $197.1 \mathrm{c}$ & $101.9 a b$ & $43.8 \mathrm{defg}$ & 45.0 cdefg \\
\hline H3 (HAV-2 × NTS-2) & 18843 defg & $62293 a b$ & 351.3 defg & $17.0 \mathrm{ab}$ & $19.7 \mathrm{c}$ & $45.5 \mathrm{fg}$ & $181.3 \mathrm{~d}$ & $74.6 \mathrm{fg}$ & $41.0 \mathrm{ij}$ & $42.5 \mathrm{~h}$ \\
\hline $\mathrm{H} 4$ (HAV-2 × EEO-2) & $20131 \mathrm{~cd}$ & $59733 \mathrm{abcd}$ & $375.4 \mathrm{~cd}$ & 15.9 bcde & $20.4 a b$ & $48.0 \mathrm{abc}$ & $185.0 \mathrm{~d}$ & $77.3 f$ & 42.3 ghij & $43.3 \mathrm{fgh}$ \\
\hline H5 (HAV-2 × HAW-1) & $17324 \mathrm{~g}$ & 43520 ghij & $322.8 \mathrm{~g}$ & $15.5 \mathrm{de}$ & 19.0 def & 46.8 cde & $200.3 \mathrm{bc}$ & $103.0 \mathrm{a}$ & 43.3 efgh & 45.3 cdef \\
\hline H6 (HSE-4 × FTT-1) & 19460 def & $62293 a b$ & 362.6 def & 15.5 de & 18.5 efg & $48.0 a b c$ & $197.4 \mathrm{c}$ & $89.7 \mathrm{de}$ & $44.3 \mathrm{cdef}$ & $45.3 \mathrm{cdef}$ \\
\hline H7 (HSE-4 × NTS-2) & $15416 \mathrm{~h}$ & 52906 def & $286.8 \mathrm{~h}$ & 16.0 bcde & $17.5 \mathrm{~h}$ & $45.3 \mathrm{~g}$ & $178.2 \mathrm{de}$ & $71.2 \mathrm{fgh}$ & 42.8 fghi & 44.0 efgh \\
\hline $\mathrm{H} 8$ (HSE-4 × EEO-2) & 19804 de & 46080 efgh & 369.0 cde & $15.6 \mathrm{cde}$ & $19.0 \mathrm{de}$ & $46.1 \mathrm{efg}$ & $209.2 \mathrm{ab}$ & $85.0 \mathrm{e}$ & 42.3ghij & $43.5 \mathrm{fgh}$ \\
\hline H9 (HSE-4 × HAW- 1 ) & 19143 def & 38400 hijk & 356.6 def & $14.8 \mathrm{efg}$ & 18.5 efg & 47.5 bcde & $184.4 \mathrm{~d}$ & $89.2 \mathrm{de}$ & 42.0 ghij & 44.3 efgh \\
\hline $\mathrm{H} 10$ (FTT-1 × NTS-2) & $18835 \mathrm{defg}$ & 61440 abc & 350.1 defg & $17.0 \mathrm{ab}$ & $19.4 \mathrm{~cd}$ & $46.8 \mathrm{cdef}$ & $207.8 \mathrm{ab}$ & $92.2 \mathrm{~cd}$ & 41.5 hij & $43.0 \mathrm{gh}$ \\
\hline H11 (FTT-1 × EEO-2) & $22364 \mathrm{~b}$ & $58880 \mathrm{bcd}$ & $416.3 \mathrm{~b}$ & $16.2 \mathrm{abcd}$ & $19.7 \mathrm{c}$ & $48.7 \mathrm{ab}$ & $213.0 \mathrm{a}$ & $87.7 \mathrm{de}$ & $41.8 \mathrm{hij}$ & $42.5 \mathrm{~h}$ \\
\hline $\mathrm{H} 12(\mathrm{FTT}-1 \times \mathrm{HAW}-1)$ & $21252 b c$ & 43520 ghij & $395.4 b c$ & $16.2 \mathrm{abcd}$ & $18.1 \mathrm{gh}$ & $49.4 \mathrm{a}$ & $195.2 \mathrm{c}$ & $101.8 \mathrm{ab}$ & $42.5 \mathrm{fghi}$ & $43.8 \mathrm{fgh}$ \\
\hline H13 (NTS-2 × EEO-2) & $15245 \mathrm{~h}$ & $37546 \mathrm{ijk}$ & $284.1 \mathrm{~h}$ & $17.3 \mathrm{a}$ & $20.0 \mathrm{bc}$ & $42.3 \mathrm{~h}$ & $185.6 \mathrm{~d}$ & $60.0 i$ & $40.5 j$ & $42.3 \mathrm{~h}$ \\
\hline H14 (NTS- $2 \times$ HAW-1) & 18408 efg & $35840 \mathrm{jk}$ & 343.4 efg & $16.9 \mathrm{abc}$ & $19.6 \mathrm{~cd}$ & 46.4 defg & $204.8 a b c$ & $107.1 \mathrm{a}$ & $42.8 \mathrm{fghi}$ & $43.8 \mathrm{fgh}$ \\
\hline H15 (EEO-2 × HAW-1) & $21907 b$ & 37546 ijk & $409.0 \mathrm{~b}$ & $16.2 \mathrm{abcd}$ & $20.8 a$ & $47.8 \mathrm{bcd}$ & $211.8 \mathrm{a}$ & $96.5 \mathrm{bc}$ & 42.0 ghij & 44.0 efgh \\
\hline \multicolumn{11}{|l|}{ Inbred parent: } \\
\hline HAV-2 & $11697 \mathrm{ij}$ & $62293 \mathrm{ab}$ & $219.3 \mathrm{ij}$ & 15.2 def & $16.2 \mathrm{ij}$ & $40.0 i$ & $138.4 \mathrm{~h}$ & $71.6 \mathrm{fgh}$ & 45.0 bcde & 46.0 bcde \\
\hline HSE-4 & $13039 i$ & $35840 \mathrm{jk}$ & $243.5 i$ & $14.1 \mathrm{fg}$ & $15.5 \mathrm{k}$ & $42.9 \mathrm{~h}$ & $166.0 \mathrm{fg}$ & $69.5 \mathrm{gh}$ & $47.3 \mathrm{a}$ & $48.5 a$ \\
\hline FTT-1 & $11001 \mathrm{j}$ & 53760 cde & $205.3 \mathrm{j}$ & $16.4 \mathrm{abcd}$ & $15.6 \mathrm{jk}$ & $40.6 i$ & $141.2 \mathrm{~h}$ & $74.7 \mathrm{fg}$ & $46.8 \mathrm{ab}$ & $48.0 \mathrm{ab}$ \\
\hline NTS-2 & $8253 \mathrm{k}$ & $60586 \mathrm{abcd}$ & $153.8 \mathrm{k}$ & $16.4 \mathrm{abcd}$ & $16.4 i$ & $35.6 \mathrm{k}$ & $146.2 \mathrm{~h}$ & $59.4 i$ & $45.3 \mathrm{bcd}$ & $46.5 \mathrm{abcd}$ \\
\hline EEO-2 & $9079 \mathrm{k}$ & 47786 efg & $169.2 \mathrm{k}$ & $14.7 \mathrm{efg}$ & $16.7 i$ & $37.5 \mathrm{j}$ & $159.5 \mathrm{~g}$ & $55.8 \mathrm{i}$ & $43.8 \mathrm{defg}$ & 44.8 defg \\
\hline HAW-1 & $8111 k$ & $34133 k$ & $151.1 \mathrm{k}$ & $13.9 \mathrm{~g}$ & $15.1 \mathrm{k}$ & $38.6 \mathrm{j}$ & $140.1 \mathrm{~h}$ & $68.2 \mathrm{~h}$ & $46.0 \mathrm{abc}$ & $47.0 \mathrm{abc}$ \\
\hline \multicolumn{11}{|l|}{ Control: } \\
\hline Hybrid 530 & $26285 a$ & 42666 ghij & $491.1 \mathrm{a}$ & $16.9 a b$ & $20.7 \mathrm{a}$ & $49.0 \mathrm{ab}$ & $195.8 \mathrm{c}$ & $74.2 \mathrm{fg}$ & $45.5 \mathrm{abcd}$ & $47.8 \mathrm{ab}$ \\
\hline Mean & 16920 & 47825 & 315.5 & 15.9 & 18.3 & 45.0 & 182.2 & 81.7 & 43.5 & 44.8 \\
\hline
\end{tabular}

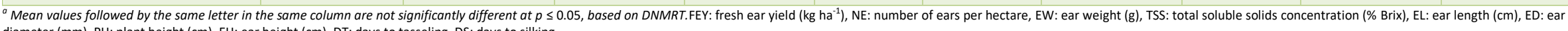
diameter ( $\mathrm{mm}$ ), PH: plant height $(\mathrm{cm})$, EH: ear height (cm), DT: days to tasseling, DS: days to silking. 
Table 3. Mean values for traits measured on 15 sweet corn hybrids, six parental inbred lines and a control variety (Hybrid 530 ), evaluated in Field 10.

\begin{tabular}{|c|c|c|c|c|c|c|c|c|c|c|}
\hline \multirow[t]{2}{*}{ Genotype } & \multicolumn{10}{|l|}{ Mean $^{a}$} \\
\hline & FEY & NE & EW & TSS & EL & ED & $\mathrm{PH}$ & $\mathrm{EH}$ & DT & DS \\
\hline \multicolumn{11}{|l|}{ Hybrid: } \\
\hline H1 (HAV-2 $\times$ HSE-4) & 15707 defg & $43520 \mathrm{abcd}$ & 292.0 defg & 16.3 bcde & 17.4 efgh & $43.6 \mathrm{abcd}$ & $168.1 \mathrm{fg}$ & 71.6 defgh & 47.8 bcde & 49.3 bcdef \\
\hline $\mathrm{H} 2$ (HAV-2 × FTT-1) & 18379 bcde & $52906 a b c$ & 344.5 bcde & 16.7 bcde & 18.1 cdefg & $45.9 \mathrm{abc}$ & 172.8 def & $82.7 \mathrm{abcd}$ & 46.3 cdef & 48.3 cdef \\
\hline H3 (HAV-2 × NTS-2) & $16240 \mathrm{cdef}$ & 58026 a & 303.7 cdef & $17.8 \mathrm{bc}$ & 18.2 cdefg & $43.2 \mathrm{abcd}$ & $183.8 \mathrm{bcde}$ & 72.4 cdefg & $46.8 \mathrm{cde}$ & 48.3 cdef \\
\hline H4 (HAV-2 × EEO-2) & $20177 a b c$ & $51200 \mathrm{abcd}$ & $377.1 \mathrm{abc}$ & 16.4 bcde & $19.6 \mathrm{abc}$ & $45.8 \mathrm{abcd}$ & 172.7 def & 65.2 ghi & 47.8 bcde & 49.5 bcdef \\
\hline H5 (HAV-2 $\times$ HAW-1) & $19867 \mathrm{abcd}$ & $43520 \mathrm{abcd}$ & $371.2 \mathrm{abc}$ & $17.7 \mathrm{bc}$ & 18.4 cdefg & $45.3 \mathrm{abcd}$ & $194.2 \mathrm{bc}$ & $90.4 \mathrm{a}$ & 44.5 ef & 46.5 efg \\
\hline H6 (HSE-4 × FTT-1) & $19707 \mathrm{abcd}$ & $52053 a b c$ & $368.9 \mathrm{abcd}$ & 16.5 bcde & 18.6 cdef & $45.5 \mathrm{abcd}$ & 192.9 bc & $85.1 \mathrm{abc}$ & 46.0 cdef & 47.8 cdefg \\
\hline H7 (HSE-4 × NTS-2) & 18417 bcde & $45226 \mathrm{abcd}$ & 348.0 abcde & $18.3 \mathrm{a}$ & $19.7 \mathrm{abc}$ & $45.2 \mathrm{abcd}$ & 185.4 bcde & 72.4 cdefg & 46.8 cde & 48.8 cdef \\
\hline H8 (HSE-4 × EEO-2) & 17117 cdef & $44373 \mathrm{abcd}$ & 320.6 bcdef & 15.7 bcde & 18.4 cdefg & 41.6 cde & $178.8 \mathrm{cdef}$ & $60.4 \mathrm{hij}$ & 47.8 bcde & 49.5 bcdef \\
\hline H9 (HSE-4 × HAW-1) & 18452 bcde & $38400 \mathrm{~cd}$ & 345.3 bcde & $15.2 \mathrm{de}$ & $18.7 \mathrm{cde}$ & $44.7 \mathrm{abcd}$ & 179.8 cdef & 78.7 abcdef & $49.5 \mathrm{abcd}$ & $50.5 \mathrm{abcd}$ \\
\hline H10 (FTT-1 × NTS-2) & $19100 \mathrm{abcd}$ & $55466 a b$ & $359.5 \mathrm{abcd}$ & $18.0 \mathrm{ab}$ & 18.4 cdefg & $46.2 \mathrm{ab}$ & 178.3 cdef & 68.5 efgh & 47.0 cde & $48.5 \mathrm{cdef}$ \\
\hline H11 (FTT-1 × EE0-2) & $21356 a b$ & $52906 a b c$ & $395.9 \mathrm{ab}$ & $17.5 \mathrm{bcd}$ & 19.1 bcde & $46.3 \mathrm{a}$ & $197.8 \mathrm{ab}$ & 76.1 bcdef & $42.0 \mathrm{f}$ & $44.0 \mathrm{~g}$ \\
\hline H12 (FTT-1 × HAW-1) & $20393 \mathrm{abc}$ & $45226 \mathrm{abcd}$ & $381.5 \mathrm{abc}$ & 16.5 bcde & 17.6 defgh & $47.4 \mathrm{a}$ & $193.9 \mathrm{bc}$ & $87.7 \mathrm{ab}$ & 45.5 def & $47.3 \mathrm{defg}$ \\
\hline H13 (NTS-2 × EEO-2) & $19048 \mathrm{abcd}$ & $46080 \mathrm{abcd}$ & $356.1 \mathrm{abcd}$ & 17.1 bcde & $20.6 \mathrm{ab}$ & $43.8 \mathrm{abcd}$ & 179.4 cdef & $64.1 \mathrm{ghi}$ & 45.3 def & 47.3 defg \\
\hline H14 (NTS- $2 \times$ HAW- 1 ) & 18519 abcde & $36693 d$ & 351.6 abcde & 16.3 bcde & 19.0 bcde & $45.0 \mathrm{abcd}$ & 181.6 cdef & 80.5 abcde & 47.5 cde & 49.8 abcdef \\
\hline H15 (EE0-2 × HAW-1) & $22623 a$ & $42666 \mathrm{abcd}$ & 424.7 a & $17.4 \mathrm{bcd}$ & $20.9 a$ & $47.2 \mathrm{a}$ & $211.4 \mathrm{a}$ & $87.4 \mathrm{ab}$ & $44.3 \mathrm{ef}$ & $46.0 \mathrm{fg}$ \\
\hline \multicolumn{11}{|l|}{ Inbred parent: } \\
\hline HAV-2 & 11940 ghij & $55466 \mathrm{ab}$ & 223.8 ghij & 17.1 bcde & $16.3 \mathrm{~h}$ & 37.7 ef & $154.6 \mathrm{gh}$ & 68.7 efgh & $49.5 \mathrm{abcd}$ & $51.3 \mathrm{abcd}$ \\
\hline HSE-4 & 14752 efgh & $42666 \mathrm{abcd}$ & 276.4 efgh & 16.3 bcde & $16.9 \mathrm{fgh}$ & $41.6 \mathrm{cde}$ & $188.1 \mathrm{bcd}$ & $90.0 \mathrm{a}$ & 47.8 bcde & 49.3 bcdef \\
\hline FTT-1 & $13527 \mathrm{fghi}$ & $48640 \mathrm{abcd}$ & 253.2 fghi & 15.6 cde & $16.4 \mathrm{~h}$ & $41.4 \mathrm{de}$ & 171.5 ef & $82.1 \mathrm{abcd}$ & $50.3 \mathrm{abc}$ & $52.0 \mathrm{abc}$ \\
\hline NTS-2 & $10047 \mathrm{ij}$ & $42666 \mathrm{abcd}$ & $188.0 \mathrm{ij}$ & $17.7 b c$ & $16.8 \mathrm{gh}$ & $34.8 \mathrm{f}$ & $148.9 \mathrm{~h}$ & $50.8 \mathrm{j}$ & 47.8 bcde & 49.3 bcdef \\
\hline EEO-2 & 11244 hij & $44373 \mathrm{abcd}$ & 209.9 hij & $15.2 \mathrm{de}$ & $16.0 \mathrm{hi}$ & $35.6 \mathrm{f}$ & $151.9 \mathrm{~h}$ & $54.3 \mathrm{ij}$ & $49.8 \mathrm{abcd}$ & $51.0 \mathrm{abcd}$ \\
\hline HAW-1 & $8393 j$ & $41813 \mathrm{bcd}$ & $158.6 \mathrm{j}$ & $15.2 \mathrm{de}$ & $14.7 i$ & $33.8 \mathrm{f}$ & $143.7 \mathrm{~h}$ & $68.6 \mathrm{efgh}$ & $52.5 \mathrm{a}$ & $53.8 \mathrm{a}$ \\
\hline \multicolumn{11}{|l|}{ Control: } \\
\hline Hybrid 530 & $19179 \mathrm{abcd}$ & $52053 \mathrm{abc}$ & $358.7 \mathrm{abcd}$ & 15.9 cde & $19.3 \mathrm{bcd}$ & $42.8 \mathrm{bcd}$ & 175.7 def & $65.9 \mathrm{fghi}$ & $52.3 \mathrm{ab}$ & $53.3 \mathrm{ab}$ \\
\hline Mean & 17008 & 47088 & 318.6 & 16.7 & 18.1 & 42.8 & 177.5 & 73.8 & 47.4 & 49.1 \\
\hline
\end{tabular}

diameter (mm), PH: plant height (cm), EH: ear height (cm), DT: days to tasseling, DS: days to silking.

Table 4. Mean squares in analysis of variance on sweet corn hybrids from a $6 \times 6$ diallel cross among inbred lines (Griffing's Method 4), for traits measured in Field 2 .

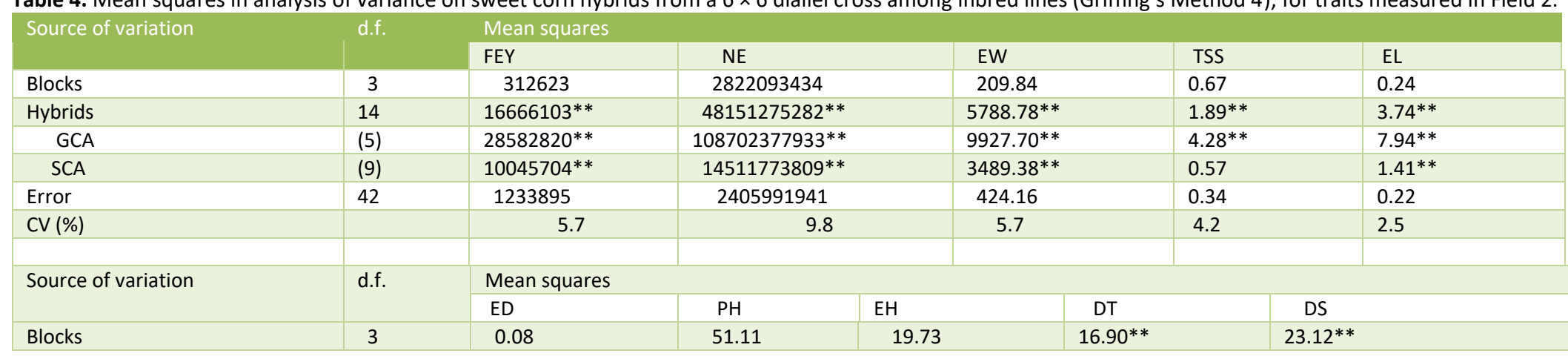




\begin{tabular}{|c|c|c|c|c|c|c|}
\hline Hybrids & 14 & $11.68^{* *}$ & $695.83^{* *}$ & $697.16 * *$ & $4.34^{*}$ & $4.05^{*}$ \\
\hline GCA & (5) & $25.21^{* *}$ & $1132.74 * *$ & $1422.28 * *$ & $7.81^{*}$ & $8.34 *$ \\
\hline SCA & (9) & $4.16^{* *}$ & $453.10 * *$ & $294.31 * *$ & 2.42 & 1.67 \\
\hline Error & 42 & 0.43 & 38.91 & 16.47 & 1.50 & 1.69 \\
\hline CV (\%) & & 1.4 & 3.2 & 4.6 & 3.8 & 4.0 \\
\hline
\end{tabular}

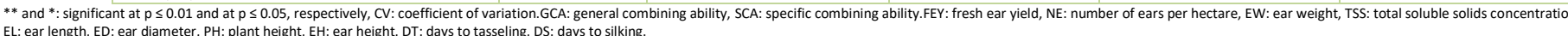

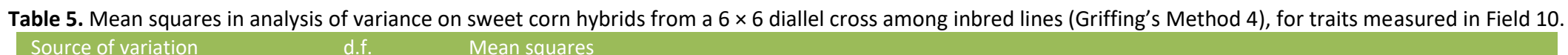

\begin{tabular}{|c|c|c|c|c|c|c|}
\hline \multirow[t]{2}{*}{ Source of variation } & \multirow[t]{2}{*}{ d.f. } & \multicolumn{5}{|l|}{ Mean squares } \\
\hline & & FEY & $\mathrm{NE}$ & EW & TSS & EL \\
\hline Blocks & 3 & 5991902 & 10382197194 & 2059.31 & $7.74^{*}$ & 0.20 \\
\hline Hybrids & 14 & $13294190 * *$ & $15506719644^{*}$ & $4641.52 * *$ & $5.13^{*}$ & $3.98 * *$ \\
\hline GCA & (5) & $26732440^{* *}$ & $34768062687^{*}$ & $9193.44^{* *}$ & 4.60 & $7.30 * *$ \\
\hline SCA & (9) & 5828496 & 4805973509 & 2112.69 & 5.44 & $2.14^{* *}$ \\
\hline Error & 42 & 2840760 & 6914683871 & 1021.97 & 2.49 & 0.24 \\
\hline CV (\%) & & 9.2 & 17.9 & 9.3 & 13.0 & 2.6 \\
\hline \multirow[t]{2}{*}{ Source of variation } & \multirow[t]{2}{*}{ d.f. } & \multicolumn{5}{|l|}{ Mean squares } \\
\hline & & ED & $\mathrm{PH}$ & $\mathrm{EH}$ & DT & DS \\
\hline Blocks & 3 & $11.32 *$ & $410.29 *$ & $253.16^{*}$ & $38.86^{*}$ & $39.24 *$ \\
\hline Hybrids & 14 & $9.58^{*}$ & $522.39 * *$ & $352.07 * *$ & $13.32 *$ & $11.33^{*}$ \\
\hline GCA & (5) & $16.60 *$ & $682.47^{*}$ & $750.18^{* *}$ & 17.45 & 14.77 \\
\hline SCA & (9) & 5.69 & $433.46^{*}$ & 130.90 & 11.04 & 9.43 \\
\hline Error & 42 & 3.79 & 115.93 & 70.44 & 6.51 & 5.70 \\
\hline CV (\%) & & 4.6 & 6.3 & 11.9 & 6.7 & 5.9 \\
\hline
\end{tabular}

6.7

5.9

Table 6. Estimates of GCA effects revealed by six sweet corn inbred lines involved in a $6 \times 6$ diallel cross (Griffing's Method 4), for traits measured in a) Field 2 and b) Field 10 .

\begin{tabular}{|c|c|c|c|c|c|c|c|c|c|c|}
\hline \multirow[t]{2}{*}{ Parent } & \multicolumn{10}{|l|}{ GCA effects } \\
\hline & FEY & NE & EW & TSS & EL & ED & $\mathrm{PH}$ & $\mathrm{EH}$ & DT & DS \\
\hline \multicolumn{11}{|c|}{ a) Field 2: } \\
\hline HAV-2 & $-497.45^{*}$ & $68266.67^{* *}$ & -8.71 & -0.09 & $-1.08^{* *}$ & -0.74 & $-9.75^{* *}$ & 0.54 & 0.48 & 0.48 \\
\hline HSE-4 & $-784.78 *$ & -14933.33 & $-14.61^{*}$ & $-0.68 * *$ & $-1.58 * *$ & -0.16 & $-8.39 * *$ & $-4.89 * *$ & 0.73 & 0.73 \\
\hline FTT-1 & $1415.89 * *$ & $106666.67^{* *}$ & $26.09 * *$ & 0.01 & $0.99 * *$ & $2.64^{*}$ & $9.10 * *$ & $8.00 * *$ & 0.42 & 0.10 \\
\hline NTS-2 & $-2043.78^{* *}$ & -2133.33 & $-38.41 * *$ & $0.88^{* *}$ & $-1.37 * *$ & 0.02 & $-4.12 *$ & $-9.05^{* *}$ & $-0.89 *$ & $-0.90 *$ \\
\hline EEO-2 & $1132.22^{* *}$ & $-27733.33^{*}$ & $21.14^{* *}$ & $0.15^{*}$ & $2.14^{* *}$ & $-2.23^{*}$ & $7.61^{* *}$ & $-8.70 * *$ & $-0.83^{*}$ & $-0.90 *$ \\
\hline HAW-1 & $777.89 *$ & $-30133.33^{* *}$ & $14.49 *$ & $-0.28 *$ & $0.90 * *$ & 0.48 & $5.55^{* *}$ & $14.10 * *$ & 0.10 & 0.48 \\
\hline \multicolumn{11}{|c|}{ b) Field 10: } \\
\hline HAV-2 & $-1166.11^{*}$ & 32711.11 & $-22.93^{*}$ & -0.03 & $-0.54 *$ & -0.52 & $-8.01 *$ & 0.31 & 0.38 & 0.35 \\
\hline HSE-4 & $-1408.44^{* *}$ & -31288.89 & $-26.38^{*}$ & -0.35 & $-0.79^{*}$ & $-1.54^{*}$ & -4.67 & -3.20 & $1.56^{*}$ & $1.35^{*}$ \\
\hline FTT-1 & $975.22 *$ & $56177.78^{*}$ & $17.54^{*}$ & 0.06 & 0.21 & $1.65^{*}$ & 3.01 & $4.76^{*}$ & -1.19 & -1.15 \\
\hline NTS-2 & $-927.44^{*}$ & 13511.11 & $-15.32 *$ & $1.01^{*}$ & $-0.90^{*}$ & -0.68 & -3.79 & $-5.82^{*}$ & 0.44 & 0.54 \\
\hline EEO-2 & 1321.89* & 2844.44 & $23.56^{*}$ & -0.22 & $1.06 * *$ & -0.33 & 4.13 & $-6.96 *$ & -1.13 & -1.02 \\
\hline HAW-1 & $1204.89 *$ & $-73955.56^{*}$ & $23.54^{*}$ & -0.49 & $0.96^{*}$ & 1.41 & $9.32 * *$ & $10.91^{* *}$ & -0.06 & -0.08 \\
\hline
\end{tabular}


able 7. Estimates of SCA effects revealed by sweet corn hybrids from a $6 \times 6$ diallel cross (Griffing's Method 4 ) among inbred lines, for traits measured in a) Field 2 and b) Field 10.

\begin{tabular}{|c|c|c|c|c|c|c|c|c|c|c|}
\hline \multirow[t]{2}{*}{ Hybrid } & \multicolumn{10}{|l|}{ SCA effects } \\
\hline & FEY & NE & EW & TSS & EL & ED & $\mathrm{PH}$ & $\mathrm{EH}$ & DT & DS \\
\hline \multicolumn{11}{|l|}{ a) Field 2: } \\
\hline $\mathrm{H} 1$ (HAV-2 $\times$ HSE-4) & 257.87 & $-102826.67^{* *}$ & 5.23 & $0.74 *$ & -0.18 & 1.30 & $-5.31 *$ & 2.69 & 0.13 & -0.03 \\
\hline $\mathrm{H} 2(\mathrm{HAV}-2 \times \mathrm{FTT}-1)$ & $-1228.13^{*}$ & -2560.00 & $-22.07^{*}$ & -0.22 & $-1.58^{* *}$ & $-2.56^{*}$ & 2.95 & $5.15^{*}$ & 0.44 & 0.60 \\
\hline H3 (HAV-2 × NTS-2) & $2399.53^{* *}$ & $55040.00 *$ & $44.54 * *$ & 0.06 & $1.91^{* *}$ & -0.25 & 0.35 & $-5.18^{*}$ & -1.00 & -0.90 \\
\hline $\mathrm{H} 4$ (HAV-2 × EEO-2) & 511.53 & $55040.00 *$ & 9.14 & -0.26 & 0.51 & 1.67 & $-7.67^{*}$ & -2.83 & 0.19 & -0.15 \\
\hline H5 (HAV-2 $\times$ HAW-1) & $-1940.80 * *$ & -4693.33 & $-36.84 * *$ & -0.31 & -0.66 & -0.16 & $9.67^{*}$ & 0.16 & 0.25 & 0.48 \\
\hline H6 (HSE-4 × FTT-1) & -155.46 & 29440.00 & -2.74 & 0.01 & 0.21 & $-2.41 *$ & 1.87 & -1.69 & 0.69 & 0.60 \\
\hline H7 (HSE-4 × NTS-2) & -739.80 & $44373.33^{*}$ & -14.06 & -0.34 & 0.27 & -1.14 & -4.11 & -3.12 & 0.50 & 0.35 \\
\hline H8 (HSE-4 × EEO-2) & 472.20 & 1706.67 & 8.66 & -0.01 & 0.56 & 1.52 & $15.18^{* *}$ & $10.33^{* *}$ & -0.06 & -0.15 \\
\hline H9 (HSE-4 × HAW-1) & 165.20 & 27306.67 & 2.91 & -0.41 & $-0.86^{*}$ & 0.74 & $-7.63^{*}$ & $-8.21^{* *}$ & $-1.25^{*}$ & -0.78 \\
\hline $\mathrm{H} 10(\mathrm{FTT}-1 \times \mathrm{NTS}-2)$ & 478.20 & 8106.67 & 8.59 & -0.02 & 0.24 & $5.91 * *$ & $8.00^{*}$ & $4.99 *$ & -0.44 & -0.03 \\
\hline $\mathrm{H} 11$ (FTT-1 × EE0-2) & 831.53 & 8106.67 & 15.21 & -0.12 & -0.26 & 0.32 & 1.49 & 0.14 & -0.25 & -0.53 \\
\hline H12 (FTT-1 × HAW-1) & 73.87 & $-43093.33^{*}$ & 1.01 & 0.36 & $1.39 *$ & -1.25 & $-14.32^{* *}$ & $-8.58^{* *}$ & -0.44 & -0.65 \\
\hline H13 (NTS-2 × EEO-2) & $2827.47 * *$ & $96426.66^{* *}$ & $52.51^{* *}$ & -0.16 & $1.68^{* *}$ & $4.35^{*}$ & $12.77^{* *}$ & $10.49 * *$ & 0.19 & -0.23 \\
\hline H14 (NTS- $2 \times$ HAW-1) & 689.53 & -11093.34 & 13.44 & 0.14 & $-0.75^{*}$ & -0.17 & $8.52^{*}$ & $13.79 * *$ & 1.13 & 0.35 \\
\hline H15 (EEO-2 × HAW-1) & $1012.20^{*}$ & 31573.33 & $19.49 *$ & 0.22 & $0.87^{*}$ & 0.84 & 3.76 & 2.84 & 0.31 & 0.60 \\
\hline \multicolumn{11}{|l|}{ b) Field 10: } \\
\hline H1 (HAV-2 × HSE-4) & -725.53 & -38400.00 & -14.76 & -0.36 & $-0.83^{*}$ & -0.35 & -3.99 & -1.70 & -0.49 & -0.53 \\
\hline $\mathrm{H} 2(\mathrm{HAV}-2 \times \mathrm{FTT}-1)$ & -437.20 & -32000.00 & -6.16 & -0.32 & 0.21 & -1.75 & -6.92 & 1.44 & 0.76 & 0.98 \\
\hline H3 (HAV-2 $\times$ NTS-2) & -673.20 & 61866.67 & -14.10 & -0.22 & -0.35 & -1.09 & $10.85^{*}$ & 1.65 & -0.36 & -0.71 \\
\hline $\mathrm{H} 4$ (HAV-2 $\times$ EEO-2) & 1014.80 & 4266.67 & 20.43 & -0.34 & $0.89 *$ & $2.69 *$ & -8.11 & -4.33 & 2.20 & 2.10 \\
\hline H5 (HAV-2 × HAW-1) & 821.13 & 4266.67 & 14.59 & 1.23 & 0.09 & 0.49 & 8.17 & 2.94 & -2.11 & -1.84 \\
\hline H6 (HSE-4 × FTT-1) & 1133.13 & 23466.67 & 21.69 & -0.20 & 0.17 & 0.58 & $9.78^{*}$ & $7.32^{*}$ & -0.68 & -0.53 \\
\hline H7 (HSE-4 × NTS-2) & $1746.47^{*}$ & -2133.33 & $33.63^{*}$ & $2.25^{*}$ & $1.08^{*}$ & 2.42 & $9.16^{*}$ & 5.20 & -1.55 & -1.21 \\
\hline $\mathrm{H} 8$ (HSE-4 × EEO-2) & $-1802.87^{*}$ & -0.001 & $-32.65^{*}$ & -0.72 & 0.00 & -2.17 & -5.40 & -5.63 & 1.01 & 1.10 \\
\hline H9 (HSE-4 × HAW-1) & -351.20 & 17066.67 & -7.91 & -0.98 & -0.41 & -0.49 & $-9.55^{*}$ & -5.20 & 1.70 & 1.16 \\
\hline $\mathrm{H} 10(\mathrm{FTT}-1 \times \mathrm{NTS}-2)$ & 45.47 & 12800.00 & 1.28 & -0.06 & 0.07 & 0.48 & -5.62 & -6.66 & 1.45 & 1.04 \\
\hline $\mathrm{H} 11$ (FTT-1 × EE0-2) & 52.13 & -2133.33 & -1.22 & 0.69 & -0.66 & 0.22 & 5.97 & 2.09 & -1.99 & -1.90 \\
\hline H12 (FTT-1 × HAW-1) & -793.53 & -2133.33 & -15.60 & -0.11 & 0.21 & 0.46 & -3.20 & -4.19 & 0.45 & 0.41 \\
\hline H13 (NTS-2 × EEO-2) & 353.20 & 27733.33 & 8.14 & 0.73 & 0.57 & 1.05 & 5.71 & -0.62 & 0.36 & 0.34 \\
\hline H14 (NTS- $2 \times$ HAW-1) & -765.53 & -44800.00 & -12.67 & -1.24 & -0.23 & -0.77 & -8.68 & -0.81 & 0.83 & 1.23 \\
\hline H15 (EEO-2 × HAW-1) & 1089.13 & 25600.00 & 21.58 & 1.09 & 0.34 & 0.31 & $13.26^{*}$ & $7.26^{*}$ & -0.86 & -0.96 \\
\hline
\end{tabular}

$* *$ and *: significant at $p \leq 0.01$ and at $p \leq 0.05$, respectively. SCA: specific combining ability, FEY: fresh ear yield, NE: number of ears per hectare, EW: ear weight, TSS: total soluble solids concentration, EL: ear length, ED: ear diameter, PH: plant height, EH: ear height, DT: days to 
Table 8. Estimates of GCA to SCA variance ratios, heterosis and heritability for traits measured on sweet corn hybrids from a $6 \times 6$ diallel cross among inbred lines, evaluated in a) Field 2 and b) Field 10.

\begin{tabular}{|c|c|c|c|c|c|c|c|c|c|c|}
\hline \multirow{2}{*}{$\begin{array}{l}\text { Genetic } \\
\text { parameter }\end{array}$} & \multicolumn{10}{|l|}{ Trait } \\
\hline & FEY & $\mathrm{NE}$ & EW & TSS & EL & ED & $\mathrm{PH}$ & $\mathrm{EH}$ & DT & DS \\
\hline \multicolumn{11}{|c|}{ a) Field 2: } \\
\hline$\sigma^{2}$ gca $/ \sigma^{2}$ sca & 0.53 & 1.95 & 0.53 & 4.01 & 1.45 & 0.06 & 0.41 & 1.01 & 1.47 & -83.41 \\
\hline $\mathrm{MPH}$ & 88.70 & 2.40 & 88.40 & 7.70 & 16.40 & 23.80 & 31.40 & 32.50 & -7.10 & -6.40 \\
\hline $\mathrm{BPH}$ & 69.70 & -10.30 & 69.30 & 3.50 & 10.70 & 18.00 & 26.20 & 24.30 & -8.70 & -8.00 \\
\hline$h^{2}{ }_{B}$ & 75.77 & 82.62 & 75.97 & 53.69 & 83.36 & 44.36 & 80.85 & 91.18 & 32.15 & 25.95 \\
\hline$h^{2} N$ & 47.99 & 76.44 & 47.99 & 76.59 & 71.54 & 8.50 & 42.86 & 65.71 & 52.77 & 66.65 \\
\hline \multicolumn{11}{|c|}{ b) Field 10: } \\
\hline$\sigma^{2}$ gca $/ \sigma^{2} s c a$ & 1.75 & -3.55 & 1.62 & -0.07 & 4.33 & 1.31 & 0.20 & 2.56 & 0.35 & 0.36 \\
\hline $\mathrm{MPH}$ & 65.90 & 2.70 & 65.90 & 6.80 & 7.20 & 22.10 & 16.20 & 11.60 & -6.60 & -5.80 \\
\hline $\mathrm{BPH}$ & 47.90 & -3.10 & 48.00 & 1.80 & 3.20 & 15.90 & 10.00 & -0.80 & -8.50 & -7.70 \\
\hline$h^{2}{ }_{B}$ & 47.92 & 23.70 & 46.96 & 21.05 & 47.94 & 18.43 & 46.71 & 49.99 & 20.75 & 19.84 \\
\hline$h^{2} N$ & 64.20 & 75.71 & 62.63 & 68.33 & 74.58 & 38.58 & 22.31 & 70.29 & 22.50 & 22.04 \\
\hline
\end{tabular}

$\sigma^{2} \mathrm{gca} / \sigma^{2}$ sca : ratio of general to specific combining ability variance, MPH: mid-parent heterosis, BPH: better-parent heterosis, $\mathrm{h}^{2} \mathrm{~N}:$ narrow-sense heritability, and $\mathrm{h}^{2}{ }_{\mathrm{B}}$ : broad-sense heritability. FEY: fresh ear yield, NE: number of ears per hectare, EW: ear weight, TSS: total soluble solids concentration, EL: ear length, ED: ear diameter, PH: plant height, EH: ear height, DT: days to tasseling, DS: days to silking.

\section{Materials and Methods}

\section{Plant materials and field procedures}

Six homozygous locally developed tropical sweet corn inbred lines were used as parents to produce $F_{1}$ single-cross hybrid progenies evaluated in this study (Table 1). The inbred lines were grown in Mar to July 2018, in Serdang, Selangor, Malaysia, where planting dates were adjusted based on previous flowering records, to ensure synchronization of tasseling and silking. Seeds of the inbred lines were planted manually at a planting density of $1.00 \mathrm{~m} \times 0.25 \mathrm{~m}$, in plots comprising of two $4-m$ long rows. Crosses in a $6 \times 6$ half-diallel manner among the inbred lines were made through hand pollinations, following the technique proposed by Russell and Hallauer (1980). At the same time, self-pollinations were also conducted manually to multiply seeds of the parental lines.

Fifteen $F_{1}$ hybrids, six parental inbred lines and one control variety, Hybrid 530, were evaluated in a randomized complete block design with three replications, in Field 2, Universiti Putra Malaysia (UPM) in early Aug to mid Oct 2018, and in Field 10, UPM, in late Aug to early Nov 2018. Field 2 is located at $3^{\circ} 00^{\prime}$ 33.0" North; $101^{\circ} 42^{\prime} 16.2^{\prime \prime}$ East, while Field 10 is located at $2^{\circ}$ 59' 28.6" North; $101^{\circ} 42^{\prime} 48.5^{\prime \prime}$ East. The Field 2 site possesses soil of Serdang series (Order Ultisol, Sub-order Udult, Typic Kandiudult) with sandy loam texture, while the Field 10 site has soil of Bungor series (Order Ultisol, Sub-order Udult, Typic Kandiudult) with loamy texture. Each genotype was planted in a five meter-long five row-plot, with a planting density of $0.75 \mathrm{~m}$ $x 0.25 \mathrm{~m}$. All experimental plots were subjected to uniform cultural practices. Before planting, the experimental plots were ploughed twice to a depth of $15 \mathrm{~cm}$ to $30 \mathrm{~cm}$, followed by soil rotorvation. The soil $\mathrm{pH}$ was increased to $5.5-6.5$ by applying ground magnesium limestone at the rate of $2 \mathrm{t} \mathrm{ha}^{-1}, 30$ days before planting. Planting was done manually at the rate of two seeds per point. The plants were subsequently thinned to just one plant per point at 10 days after planting. Fertilizers were applied at the rate of $140 \mathrm{~kg} \mathrm{ha}^{-1} \mathrm{~N}, 100 \mathrm{~kg} \mathrm{ha}^{-1} \mathrm{P}_{2} \mathrm{O}_{5}$, and $120 \mathrm{~kg}$ $\mathrm{ha}^{-1} \mathrm{~K}_{2} \mathrm{O}$, using the compound fertilizer Nitrophoska Green (NPK $15: 15: 15)$ at 7 days after planting, Nitrophoska Blue (NPK $12: 12: 17)$ at 28 days after planting, and Urea ( $46 \% \mathrm{~N})$ in equal splits at 15 and 35 days after planting. Weeds were controlled using the pre-emergence herbicide Gesaprim 500 FW (1-Chloro3-ethylamino-5-isopropylamino-2,4,6-triazine), at the rate of 2 $\mathrm{ml} \mathrm{L}^{-1}$, immediately after planting, and the post-emergence herbicide, Basta 15 (Ammonium 2-amino-4-[hydroxyl (methyl) phosphoryl] butanoate), at the rate of $3 \mathrm{ml} \mathrm{L}^{-1}$ when required during the plant development. Sprinkler irrigation was employed throughout the plant growth period when necessary. Other details of field practices followed those of Saleh et al. (2002). Pre- and post-harvest agronomic data were taken from each plot. Only plants in the harvest area covered by the middle four meters of the middle three rows were used for data collection and sampling.

\section{Statistical and genetic analyses}

Data from each location were subjected to the analysis of variance (ANOVA) using PROC GLM in SAS (Statistical Analysis System, version 9.4), to determine the significance of variation among the genotypes. Subsequently, the Duncan's New Multiple Range Test (DNMRT) was employed to compare mean performances among the hybrids, parental inbred lines and the control variety.

Using the same SAS software, the diallel analysis was executed utilizing the DIALLEL-SAS program (Zhang and Kang, 2003), following Griffing's Method 4 (one set of $F_{\text {I }}$ progenies) for each location separately (Griffing, 1956), applying fixed-effects model (Model 1). The linear model for data at each location was as follows:

$$
Y_{i j k}=\mu+r_{k}+g_{i}+g_{j}+s_{i j}+e_{i j k}
$$

where: $Y_{i j k}$ : observed value of each experimenta1 unit, $\mu$ : mean of the population, $r_{k}$ : replication effect, $g_{i}$ : GCA effect of the $i^{\text {th }}$ parent, $g_{j}$ : GCA effect of the $j^{\text {th }}$ parent, $s_{i j}$ : SCA effects of $\mathrm{ij}^{\text {th }} \mathrm{F}_{1}$ hybrid, and $e_{i j k}$ : residual effects.

Heterosis revealed by each hybrid was calculated as the percentage of the superiority of the hybrids over its mid-parent (MPH) or better-parent (BPH), as follows:

$$
\begin{aligned}
& \mathrm{MPH}=\left(\frac{F_{1}-M P}{M P}\right) \times 100 \\
& \mathrm{BPH}=\left(\frac{F_{1}-B P}{B P}\right) \times 100
\end{aligned}
$$


where: $F_{1}$ is the hybrid value, $M P$ is the mid-parent value (average values of the two individual inbred parents involved in the cross), and $B P$ is the better-parent value.

Broad-sense heritability was estimated from the variance components from the ANOVA table in the separate individual analyses, using the standard formula proposed by Singh and Chaudhary (1979), as follows:

$$
\mathrm{h}_{\mathrm{B}}^{2}=\frac{\sigma_{\mathrm{G}}^{2}}{\sigma_{\mathrm{P}}^{2}}
$$

where: $\mathrm{h}_{\mathrm{B}}^{2}$ : broad-sense heritability, $\sigma_{\mathrm{G}}^{2}$ : genotypic variance, and $\sigma_{\mathrm{P}}^{2}$ : phenotypic variance. Estimates higher than $50 \%$ indicate a high heritability, estimates between 20 to $50 \%$ indicate a moderate heritability, while those lower than $20 \%$ indicate low heritability (McWhirter, 1979).

Narrow-sense heritability was estimated from the variance components in the ANOVA table for the analysis of combining ability. Heritability for the separate analysis was estimated following the method proposed by Rojas and Sprague (1952), using PROC VARCOMP in the same SAS software, as follows:

$$
\mathrm{h}_{\mathrm{N}}^{2}=\frac{2 \sigma_{\mathrm{G}}^{2}}{\left(2 \sigma_{\mathrm{G}}^{2}+\sigma_{\mathrm{S}}^{2}+\sigma_{\mathrm{e}}^{2}\right)}
$$

where: $h_{N}^{2}$ : narrow-sense heritability, $\sigma_{G}^{2}$ : variance resulting from GCA, $\sigma_{S}^{2}$ : variance due to SCA, and $\sigma_{e}^{2}$ : variance due to residual error.

\section{Conclusion}

Hybrids $\mathrm{H} 11, \mathrm{H} 12$ and $\mathrm{H} 15$ were found to be the most superior new hybrids, as reflected by their performance as well as magnitudes of combining abilities and heterosis revealed by their parental lines for almost all traits measured at both locations. Consistently high magnitudes of mid- and betterparent heterosis revealed were indications of good accumulation of diverse favourable dominant additive genes in their parents. The inbred lines FTT-1, EEO-2 and HAW-1 had high positive GCA effects for fresh ear yield and most of the important yield components. The cross combinations, HAV- $2 \times$ NTS-2 (H3), HSE-4 $\times$ NTS-2 (H7), NTS-2 $\times$ EEO-2 (H13) and EEO-2 $\times$ HAW-1 $(\mathrm{H} 15)$ revealed high positive SCA effects for these important traits at each location. Both additive and nonadditive gene actions were found important in the genetic control of most traits in the populations, although additive gene actions were more prevalent. The promising hybrids should be further tested in large scale trials, before any of them could be released as new cultivars.

\section{Acknowledgements}

The authors wish to thank Universiti Putra Malaysia and the Ministry of Higher Education of Malaysia, for the financial support to enable the execution of this research through the Geran Putra Berimpak Research Grant Scheme under the Project UPM/700-2/1/GPB/2017/9549500.

\section{References}

Ahmad S, Quamruzzaman AKM, Nazim UM (2009) Combining ability estimates of tomato (Solanum lycopersicum) in late summer. SAARC J Agric. 7:43-56.

Alan Ö, Kinaci G, Kinaci E, Kutlu I, Basciftci ZB, Sonmez K, Evrenosoglu $Y$ (2013) Genetic variability and association analysis of some quantitative characters in sweet corn. Not Bot Horti Agrobot Cluj Napoca. 41:404-413.

Ali ES, Saleh GB, Wahab ZB, Rahim AA (2003) Performance, heritability and correlation studies on varieties and population cross of sweet corn. Asian J Plant Sci. 2:756-760.

Azad AKM, Biswas BK, Alam N, Alam SS (2014) Combining ability and heterosis for some quantitative traits in experimental maize hybrids. Plant Breed Seed Sci. 70:41-54.

Bordallo PDN, Pereira MG, Amaral Júnior ATD, Gabriel APC (2005) Diallel analysis of sweet and regular corn genotypes for agronomic characters and total protein content. Hortic Brasileira 23:123-127 (in Portuguese, with abstract in English).

Burt AJ, Grainger CM, Shelp BJ, Lee EA (2011) Heterosis for carotenoid concentration and profile in maize hybrids. Genome. 54:993-1004.

Dermail A, Bhalang S, Sompong C, Jirawat S, Kamol L (2019) Hybrid prediction based on SSR-genetic distance, heterosis and combining ability on agronomic traits and yields in sweet and waxy corn. Sci Hortic. 259:1-12.

Dhasarathan M, Babu C, Iyanar K (2015) Combining ability and gene action studies for yield and quality traits in baby corn (Zea mays L.). SABRAO J Breed Genet. 47:60-69.

Falconer DS, Mackay TF (1996) Introduction to quantitative genetics. Pearson/Prenctice Hall, New York, NY, USA.

Griffing B (1956) Concept of general and specific combining ability in relation to diallel crossing systems. Aust J Biol Sci. 9:463-493.

Hallauer AR, Miranda FJB (1988) Quantitative Genetics in Maize Breeding, lowa State University Press, Ames, IA, USA.

Ha V (1999) Genetic analysis of some yield components and kernel quality in sweet corn. Rom Agric Res. 11-12:9-20.

Kashiani P, Saleh G, Osman M, Habibi D (2011) Sweet corn yield response to alternate furrow irrigation methods under different planting densities in a semi-arid climatic condition. Afr J Agric Res. 6:1032-1040.

Khanduri A, Prasanna BM, Hossain F, Lakhera PC (2010) Genetic analyses and association studies of yield components and kernel sugar concentration in sweet corn. Indian J Genet Plant Breed. 70:257-263.

Kumar S, Uttam C, Satish KG, Devlash R (2019) Combining ability and heterosis for yield contributing and quality traits in medium maturing inbred lines of maize (Zea mays L.) using line $x$ tester. Int J Chem Stud. 7:2027-2034.

Le Gouis J, Beghin D, Heumez E Pluchard P (2002) Diallel analysis of winter wheat at two nitrogen levels. Crop Sci. 42:11291134.

McWhirter KS (1979) Breeding of cross-pollinated crops. In: Knight R (ed) Plant breeding. Australian Vice-chancellors' Committee. p.79-121.

Revilla P, Abuin MC, Malvar RA, Soengas $P$, Ordás B, Ordás A (2005) Genetic variation between Spanish and American versions of sweet corn inbred lines. Plant Breed. 124:268-271. 
Rojas BA, Sprague GF (1952) A comparison of variance components in corn yield trials: III. General and specific combining ability and their interaction with locations and years. Agron J. 44:462-466.

Rovaris SRS, André LBO, Eduardo S, Paulo BG, Maria EAGZP (2017) Genetic parameter estimates and identification of superior white maize populations. Acta Scientiarum Agron. 39:157-164.

Russell WA, Hallauer AR (1980) Corn. In: Fehr WR, Hadley HH (eds) Hybridization of crop plants. Am Soc Agron and Crop Sci Soc Am., Madison, WI, USA. p.299-312.

Saleh GB, Alawi SAS, Panjaitan K (2002) Performance, correlation and heritability studies on selected sweet corn synthetic populations. Pak J Biol Sci. 5:251-254.

Singh J, Chaudhary LB (1979) Biometrical methods in quantitative genetic analysis, Kalyani Publishers, New Delhi, India.

Sujiprihati S, Saleh GB, Ali ES (2003) Heritability, performance and correlation studies on single cross hybrids of tropical maize. Asian J Plant Sci. 2:51-57.
Suzukawa AK, Carolina BP, Mayara MG, Rodrigo IC, Douglas MZ, Marlon MD, Carlos AC (2018) Diallel analysis of tropical and temperate sweet and supersweet corn inbred lines. Revista Ciência Agronômica. 49:607-615.

Suzuki DT, Griffiths AJF, Lewontin RC (1981) An introduction to genetic analysis, WH Freeman and Company, San Francisco, CA, USA.

Vacaro E, Neto B, Fernards J, Pegoraro DJ, Nuss CN, Conceicao LD (2002) Combining ability of twelve maize populations. Pesquisa Agropecuária Brasileira. 37:67-72.

Yao W, Zhang B, Yin X, Zhang P, Huang Y (2018) Genetic combining ability analysis of soluble sugar content in supersweet corn. Southwest China J Agric Sci. 31:1116-1121 (in Chinese, with abstract in English).

Zhang Y, Kang MS (2003) DIALLEL-SAS: A program for Griffing's diallel methods. In: Kang MS (ed) Handbook of formulas and software for plant geneticists and breeders. The Haworth Press Inc., New York, NY, USA. p.1-19. 